Brit. J. Ophthal. (1964) 48, 196.

\title{
PATHOLOGICAL FINDINGS IN A CASE OF PRIMARY CONGENITAL GLAUCOMA COMPARED WITH NORMAL INFANT EYES*
}

\author{
BY \\ J. S. SPEAKMAN AND T. S. LEESON \\ From the Departments of Ophthalmology and Anatomy, University of Toronto
}

EARLIER studies have illustrated the gross changes which take place in the configuration of the anterior chamber with increasing age (Allen, Burian, and Braley, 1955; Maumenee, 1958; Sondermann, 1930), resulting in a marked deepening of the angle. At term the whole of the trabecular meshwork, scleral spur, and a variable portion of ciliary body lie exposed in the anterior chamber. Coincidental with the deepening of the angle, the canal of Schlemm forms from the vascular plexus in the region of the scleral spur. Interposed between the canal of Schlemm and the anterior chamber lies the trabecular meshwork, which in early foetal life consists of a solid mass of cells and fibres. By birth the meshwork appears laminated and, although it is presumed that communications exist between adjacent lamellae, these cannot be visualized in routine paraffin sections. Since paraffin sections exclusively have been employed in the past to study the angle of infant eyes, virtually nothing is known about the finer drainage channels within the meshwork. By using the microscope to examine flat preparations of the meshwork, one performs in effect a refined gonioscopy, and this makes possible a more detailed visualization of the intertrabecular spaces which are essential for the normal circulation of aqueous. A comparison of the findings in congenital glaucoma with normal eyes suggests that a defect in the development of these spaces may be responsible for obstruction to aqueous outflow.

The Table (opposite) records clinical data concerning the cases used in this study. It is important to remember that the eyes which were used to establish criteria for normality were not obtained from healthy individuals. Although the eyes did not receive a detailed clinical examination, only those which were removed from a baby with a congenital corneal epithelial dystrophy were thought to be abnormal. The child with congenital glaucoma died 2 hours after a bilateral goniotomy and the eyes were fixed in osmic acid which made possible an electron microscopic examination. Conventional methods of study with meridional paraffin sections have been used to record the gross configuration of the angle. In order to visualize channels running through the meshwork, thin flat teased preparations were removed at different levels with jeweller's forceps. This technique avoids the artefacts of shrinkage and distortion associated with paraffin sectioning, but introduces artefacts of its own due to tissue disruption. It is important to examine preparations that are not too thin in order that normal tissue relationships are preserved.

* Received for publication September 16, 1963. 
TABLE

CLINICAL DATA OF SIX CASES EXAMINED

\begin{tabular}{c|c|c|c}
\hline Age (mths) & Sex & Interval Before Fixation (hrs) & \multicolumn{1}{|c}{ Clinical History } \\
\hline 5 & Female & 12 (approx.) & Hydrocephalus \\
\hline 18 & Male & 12 (approx.) & $\begin{array}{l}\text { Gastroenteritis } \\
\text { Epithelial corneal dystrophy }\end{array}$ \\
\hline 3 & Female & $\frac{1}{2}$ & $\begin{array}{l}\text { Premature 1 mth } \\
\text { Bronchopeumonia }\end{array}$ \\
\hline 7 yrs & Male & 8 & $\begin{array}{l}\text { Mental defective } \\
\text { Simple measles }\end{array}$ \\
\hline 6 & Male & 10 & $\begin{array}{l}\text { Congenital glaucoma } \\
\text { Died 2 hrs after bilateral goniotomy }\end{array}$ \\
\hline (foetus) & $?$ & $?$ & \begin{tabular}{l} 
Therapeutic abortion for rubella \\
\hline
\end{tabular} \\
\hline
\end{tabular}

Findings in Normal Eyes

Paraffin sections demonstrate the marked deepening of the chamber angle with increasing age which results from an elongation of the innermost trabecular fibres (Fig. 1a,b). This exposes the whole of the inner aspect of the meshwork to the anterior chamber and contractions of the circular muscle fibres of the ciliary body will be transmitted to the entire meshwork.

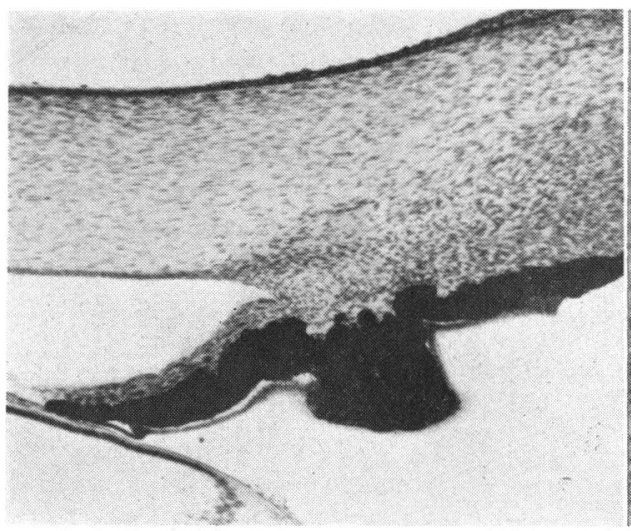

(a)

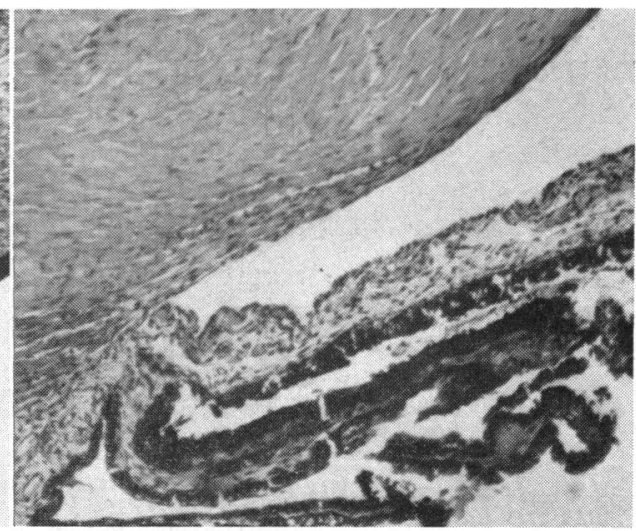

(b)

FIG. $1(a, b)$.-Paraffin sections showing normal deepening of chamber angle and lengthening of innermost trabecular fibres with increasing age. (a) Foetus $3 \mathrm{mths}$. (b) Infant $3 \mathrm{mths} . \times 40$.

Uveal Meshwork.-Flat preparations (Fig. 2a,b, overleaf) show long strands of fibres running in an antero-posterior direction from the root of the iris to Schwalbe's line. Connexions divide the spaces between these iris processes or pectinate ligaments into circular openings. Many of the spaces between fibres are obstructed by thin sheets of cytoplasm which are frequently perforated by round openings or attenuated into thin strands. In the adult the openings in the uveal meshwork are much larger and the membranes of cytoplasm between the fibres largely disappear. 


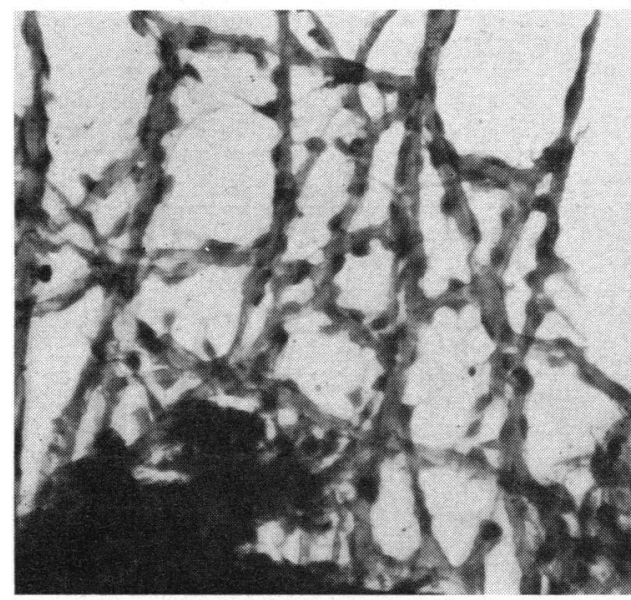

(a)

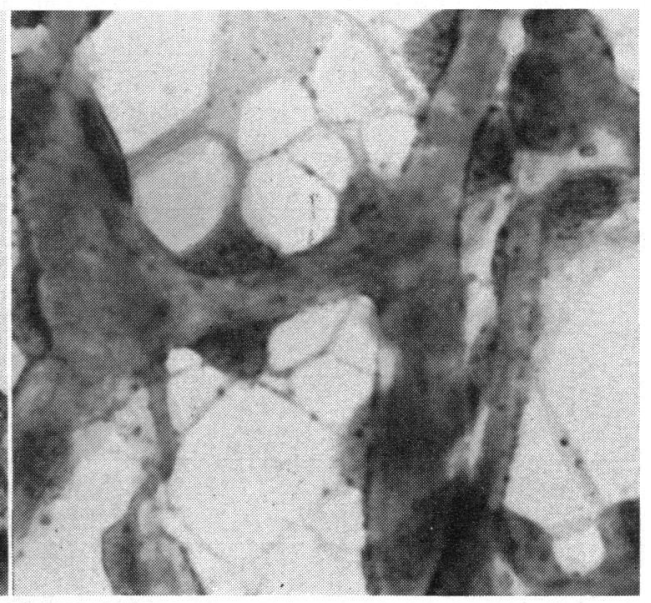

(b)

FIG. $2(a, b)$.-Flat preparations of uveal meshwork, showing perforated membranes of cytoplasm stretching across gaps between fibres. Infant $18 \mathrm{mths}$. (a) $\times 140$. (b) $\times 1250$.

Corneo-scleral Meshwork.-This can be identified because the fibre bundles form lamellae which run circumferentially, parallel to Schlemm's canal. In the innermost layers near the uveal meshwork, oval and spiral defects lead from one intertrabecular space to the next. These larger openings are confined to the layers just beneath the uveal meshwork. The outer third of the meshwork consists of a mass of cells and fibres in which no definite lamellae can be identified (Fig. $3 a-c$, opposite). In thin flat preparations the cytoplasm appears vacuolated and the spaces closely resemble the degenerative vacuoles seen in corneal endothelium. There are some quite fine spaces or vacuoles less than one micron in diameter, but the majority are larger and occasionally contain pigment or red blood cells. Careful focusing shows that the larger vacuoles communicate with each other, forming irregular tunnels through the meshwork. In the adult the smaller vacuoles are absent but one may find areas where larger vacuoles or spaces are present. In the past such areas have been called cystic or foamy degeneration of the meshwork (Ashton, 1959; Becker, 1959; Teng, Katzin and Chi, 1957) but, in view of the present findings, it seems more likely that they represent a persistence into adult life of structures which are found commonly in infancy. Flat preparations of the outermost portion of the meshwork reveal the outflow pathways which lie in the trabecular wall of Schlemm's canal (Fig. 4a,b, opposite).

The fibre bundles of collagen are not present here and the formation of spaces develops to a remarkable degree. The cytoplasm surrounds relatively enormous cavities and smaller intercommunicating spaces are present in or between every cell. Similar spaces are found in the trabecular meshwork lying immediately below the wall of the canal. The large spaces found in the 3-month-old baby were similar to those seen in adult eyes but the smaller spaces appeared to be present in greater numbers. A fine vacuolation occurred in some cells which was not seen in the adult. 


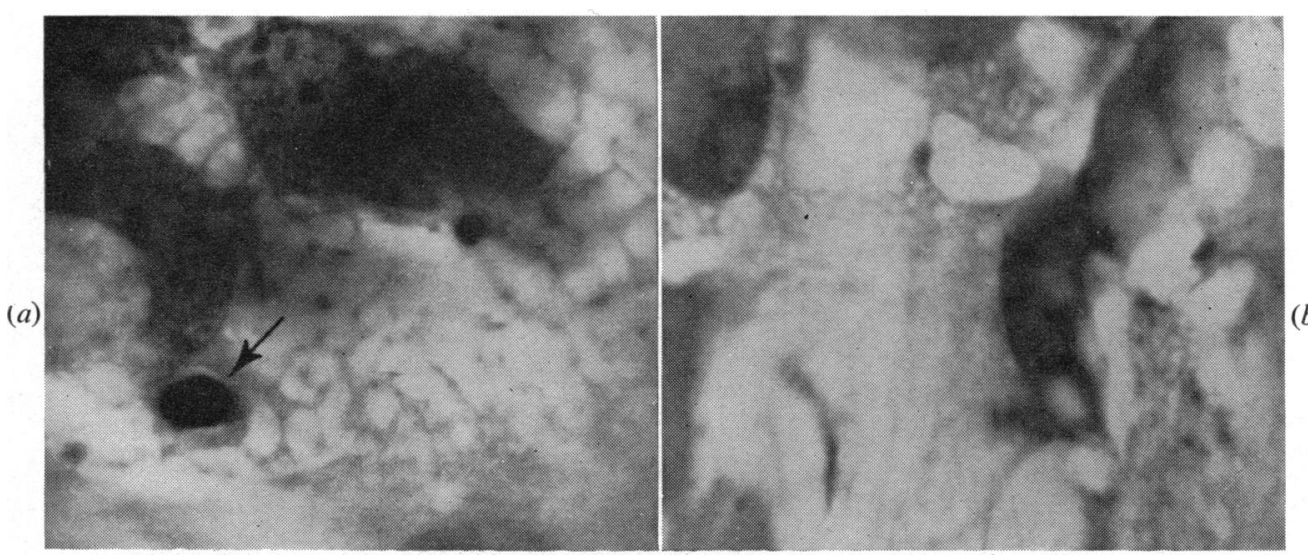

(b)

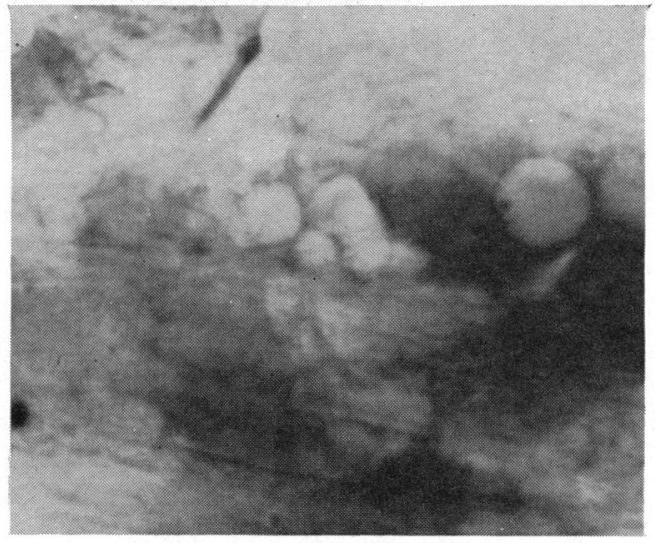

(c)

FIG. $3(a, b, c)$.-Flat preparations of corneo-scleral meshwork, showing spaces of varying sizes in cytoplasm lying between lamellae. Pigment (arrow) and red cells are seen in these spaces. (a) 5 mths. $(b)$ and $(c) 3$ mths. $\times 1250$.

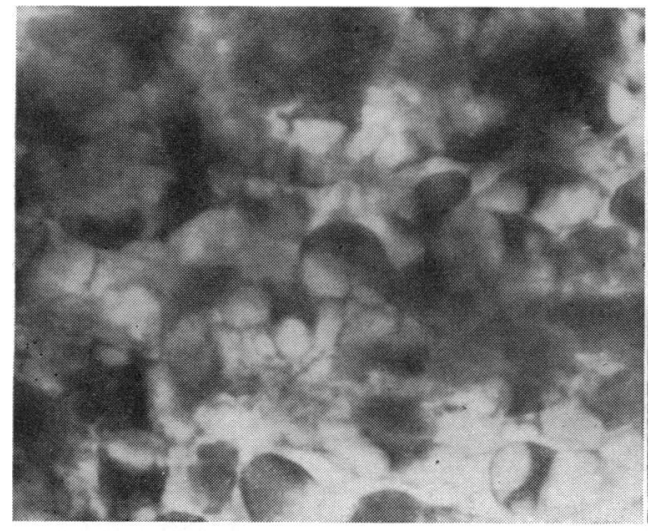

(a)

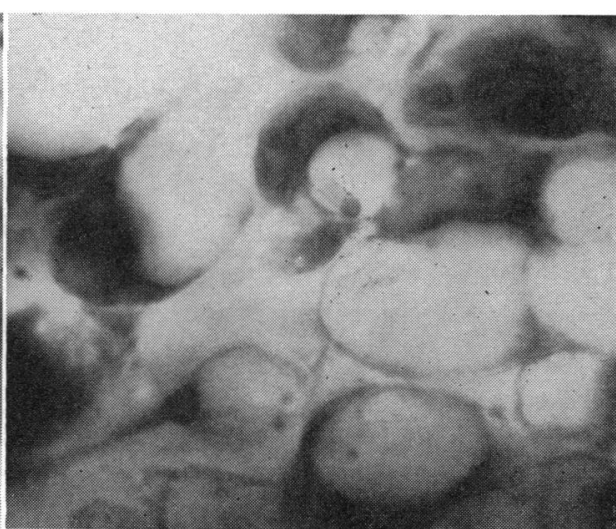

(b)

FIG. $4(a, b)$.- - Flat preparations of trabecular wall of Schlemm's canal, showing spaces in the endothelial meshwork. 3 mths. $(a) \times 625$. $(b) \times 1250$. 


\section{Findings in Congenital Glaucoma}

Meridional paraffin sections show a gross defect in the development of the angle (Fig. 5). The root of the iris is closely approximated to Schwalbe's line and the ciliary sulcus has not formed. Consequently there is a more limited exposure of the meshwork to the anterior chamber and, because of the shorter length of the uveal fibres, contraction of the circular ciliary muscle fibres will be less effective in opening the meshwork. The canal of Schlemm is rudimentary and contains red blood cells and clotted plasma; it appears compressed and is difficult to differentiate from the scleral plexus. The inner portion of the trabecular meshwork is laminated, and red blood cells are present at all levels including the trabecular wall of Schlemm's canal (Fig. 6). In some sections an eosinophilic mass occupies the middle third of the meshwork which has the same staining properties as plasma clot in the canal of Schlemm. The number of red cells which have lodged in the meshwork vary greatly in different regions of the angle. Where they are few in number they are located along a line extending from Schwalbe's line to the most anterior portion of Schlemm's canal which may indicate the pathway of greatest porosity. It could also indicate the site from which the absorption of blood is delayed the longest but the former appears more likely in view of the short interval after surgery.

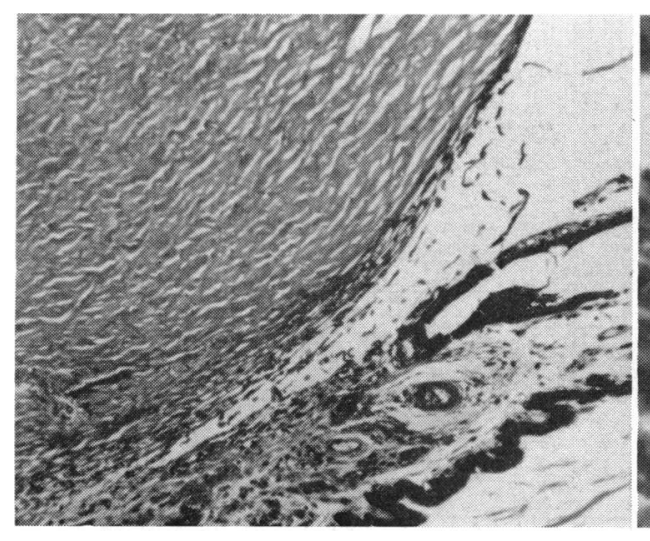

FIG. 5,-Paraffin section showing, in congenital glaucoma, marked reduction in depth of chamber angle and close approximation of root of iris to Schwalbe's line. 6 mths. $\times 40$.

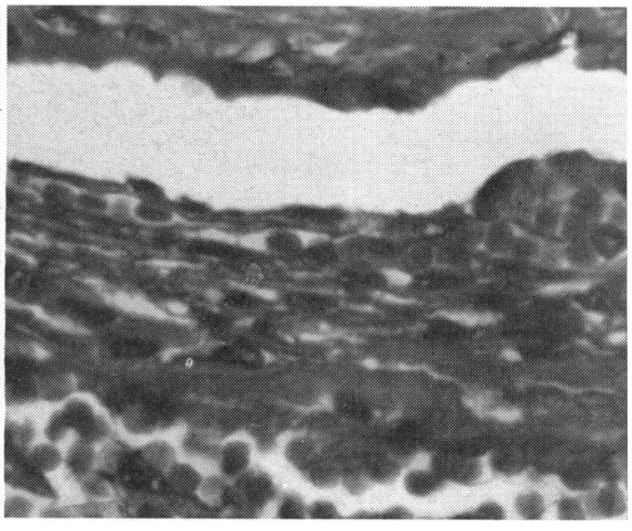

Fig. 6.-Paraffin section showing red cells and clotted plasma in outer layers of trabecular meshwork. Congenital glaucoma. $\times 625$.

Uveal Meshwork.-Flat preparations (Fig. 7, opposite) show large openings into the underlying layers but the length of the fibres running from the iris root to Schwalbe's line is greatly reduced and the pectinate ligaments are more matted together. Several layers of fibres were removed which surrounded elongated overlapping clefts (Fig. 8, opposite).

There is a noticeable reduction in cellularity in this region and the cytoplasm which covers the beams and bridges the clefts is free of vacuoles. In the outer layers of the meshwork, although larger spaces are present near some of the cell nuclei, these appear to be fewer in number, and in contrast to the normal eyes examined there are large numbers of much smaller spaces or vacuoles in the cytoplasm covering the trabecular beams (Fig. 9a,b, opposite). 


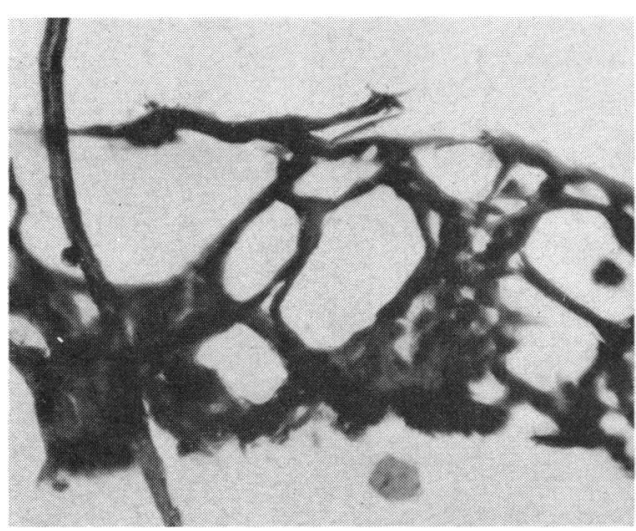

Fig. 7.-Flat preparation showing large openings in uveal meshwork between short pectinate ligaments. Congenital glaucoma. $\times 140$.

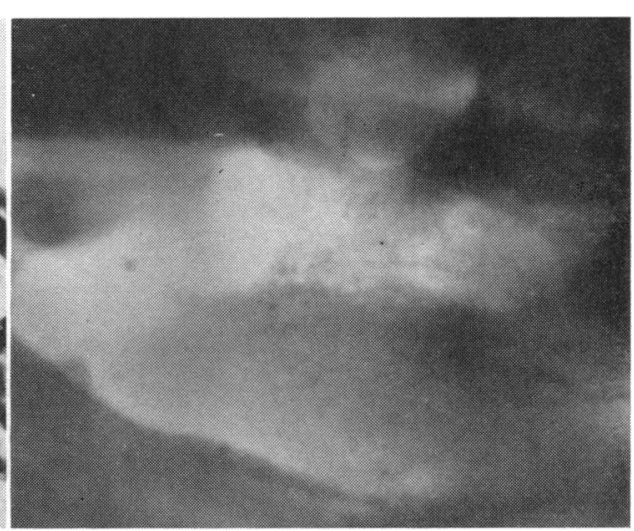

Fig. 8.-Flat preparation of inner corneo-scleral meshwork, showing large clefts and circular and spiral openings between lamellae. Congenital glaucoma. $\times 1250$.

(a)

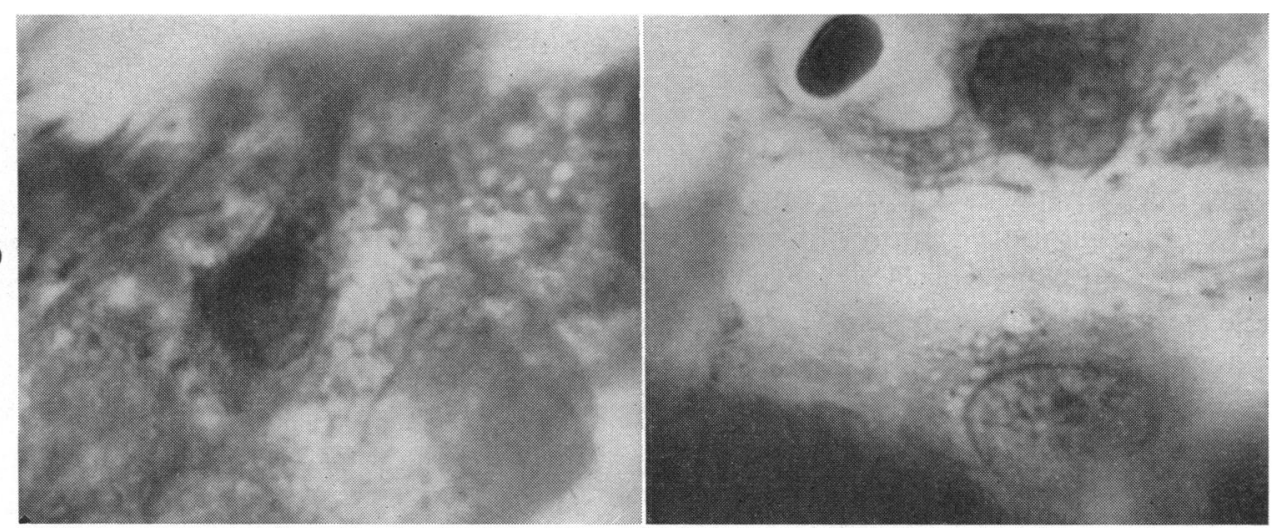

Fig. $9(a, b)$.-Flat preparations of outer corneo-scleral meshwork, showing many small vacuoles in cytoplasm. Congenital glaucoma. $\times 1250$.

Canal of Schlemm.-Clotted blood could be identified but, because the fibre bundles forming the meshwork were so tightly bound together, it was not possible to remove thin preparations in this region without causing considerable distortion of tissue. In contrast to the meshwork itself several larger spaces and numerous red blood cells are present in the region of the canal of Schlemm and some of these spaces are probably analogous to those found in the endothelial meshwork (Fig. 10, overleaf). No large spaces, comparable in size to those seen in the wall of the canal in normotensive eyes are found, and the most porous portion of the meshwork is nearer to Schwalbe's line. The structure of the outermost portion of the meshwork in the glaucomatous eye resembles more closely the tissue normally found adjacent to the scleral wall of Schlemm's canal.

Trabecular Meshwork.-Electron microscopic examination (Fig. 11, overleaf) was limited by post mortem changes, which are apparent mainly in the cellular elements. As in material from normal infants studied previously, the beams are incompletely 


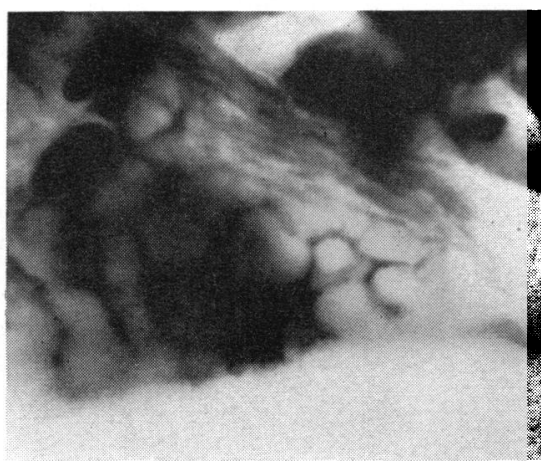

FIG. 10.-Flat preparation showing larger spaces in the region of Schlemm's canal analogous to those in the endothelial meshwork. Congenital glaucoma. $\times 975$.

Fig. 11.-Electron microphotograph showing an immature trabecular beam consisting of a core of fine fibres surrounded by a basement membrane (arrow) and a layer of cytoplasm containing many small vesicles and showing post mortem change.

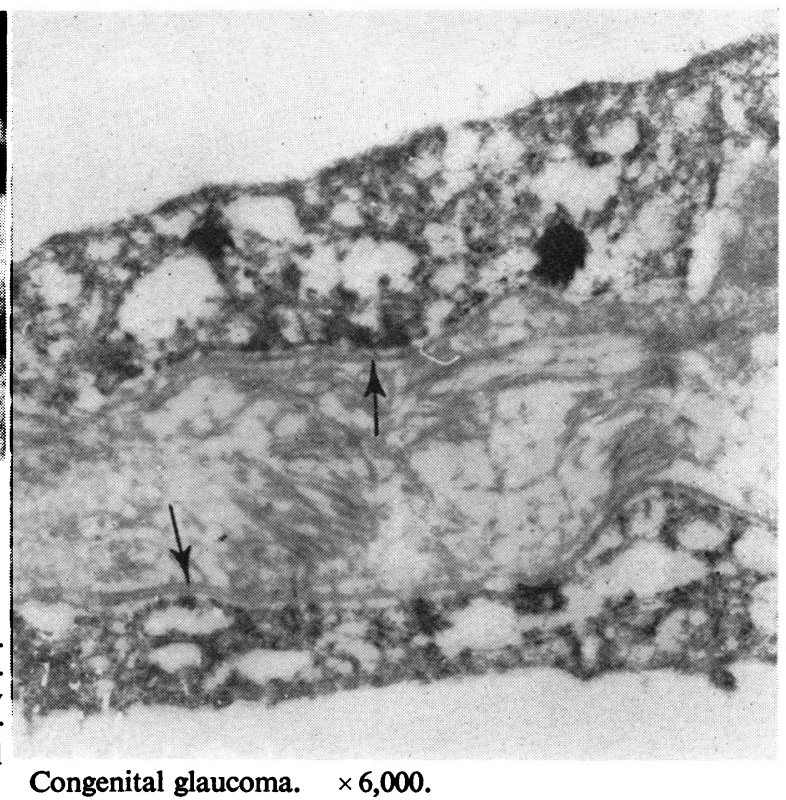

Congenital glaucoma. $\times 6,000$.

formed and the diameter is less than that found in the adult. Between the beams the intertrabecular spaces are open and occasionally contain a red blood cell. The centre of a beam is composed of unit fibres of collagen running along the length of the beam, possibly in an open spiral which was an appearance also seen by light microscopy. The core material is limited at the periphery by an extracellular basement membrane, upon which lies the attenuated cytoplasm of the endothelial cells, and there is no evidence of a clear zone. In the endothelium post mortem changes are apparent; for example, the mitochondria are distorted. There is no gross vacuolation of the cytoplasm and, although many vesicles are present, the majority are probably too small to be apparent by light microscopy. Long-spacing collagen, or curly collagen, which is normally found in the clear zone in the adult, was not evident in any of the sections examined. In this respect the eye with congenital glaucoma resembles meshwork from normal infants (Leeson and Speakman, 1961) and differs from adult cases of open-angle glaucoma in which a remarkable nodular proliferation of this material has been found (Speakman and Leeson, 1962).

\section{Discussion}

The deepening of the chamber angle and the separation of the innermost trabecular fibre bundles into lamellae are important changes which take place in the meshwork with increasing age, and can be seen in routine paraffin sections. It is not possible by this method of examination, however, to visualize the finer communications between adjacent lamellae which are apparent in flat teased preparations. These are conspicuous oval and spiral openings in the innermost layers. Near to the trabecular wall of Schlemm's canal and in the sheets of cytoplasm which bridge the openings in the uveal and corneo-scleral meshwork, there are large numbers of spaces or vacuoles which vary in size from 1 to $2 \mu$ to several times the diameter of a red blood cell. The larger spaces in the inner layers quite clearly establish communica- 
tion between adjacent lamellae and in the outer layers many of the larger spaces also intercommunicate. A fundamental problem is to ascertain if these spaces represent a post mortem change comparable to that found in the corneal endothelium (Speakman, 1959) or structures which function in the living eye. In the endothelial meshwork, where the spaces reach their maximum size, the evidence indicates that they are present in vivo. Much of the evidence supporting this view also applies to the spaces found within the meshwork itself. No difference in the appearance of the meshwork was seen in eyes from monkeys and surgical specimens of adult human eyes in which fixation has been either immediate or delayed. If vacuole formation in the corneal endothelium is prevented by rapid fixation, the cyst-like spaces in the cells of the meshwork and in the lining of Schlemm's canal persist (Speakman, 1960). The spaces may contain pigment or red blood cells, which also suggests that they represent functioning drainage channels. If the spaces were similar in origin to those found in the corneal endothelium, one would expect a more widespread involvement of the endothelial cells in the meshwork. However, the cytoplasm covering the larger beams and bridging the large clefts does not show this change. Also, if one examines the region of Schwalbe's line, there is an abrupt transition from vacuolated corneal endothelium to normal-looking trabecular endothelium. This indicates a marked difference in behaviour between the endothelium of the cornea and the meshwork. Although electron microscopic examination of the congenital glaucoma eye shows small vacuoles in the degenerate cytoplasm, these do not attain the size of the spaces seen by light microscopy and the majority would be invisible. Conversely, large spaces in the endothelial meshwork have been demonstrated in tissue which has been carefully fixed (Garron and Feeney, 1959; Holmberg, 1959). Although there may be some doubt about the nature of the very small vacuoles less than one micron in diameter, the evidence indicates that the larger spaces in the cytoplasm of the meshwork as well as those in the inner wall of Schlemm's canal are present in life.

If one accepts that the larger spaces noted in the meshwork are vital structures, what possible function can they serve? The most reasonable explanation is that they form a tortuous system of channels which conduct aqueous to Schlemm's canal from the anterior chamber. The process of canalization probably begins with the formation of small vacuoles between adjacent cells. With increasing age the vacuoles enlarge and coalesce providing channels leading from one cell to the next. If this interpretation is correct, one would expect to find more smaller spaces in the younger eyes and this is true if one compares the infant with the adult. Also one would expect the fine spaces to disappear when separation and differentiation of the trabecular lamellae is complete. When one examines the cytoplasm covering the innermost layers, the fine vacuoles are not found nor are they seen in the sheets of cytoplasm which bridge the larger clefts. These are areas in which differentiation is most advanced.

In primary congenital glaucoma it has been recognized that a developmental defect is responsible and several theories have been postulated to explain the obstruction to outflow. Some have thought that an impermeable membrane, which is visible gonioscopically, lies across the inner surface of the chamber angle. The flat preparations show clearly, however, that large openings are present in this region. 
More recently it has been suggested that an anterior insertion of the long ciliary muscle compresses the meshwork, thus reducing aqueous outflow (Maumenee, 1958, 1962). A comparison of the porosity of the meshwork in different eyes is difficult because no quantitative methods are available and the size of the drainage channels varies greatly from one area of the meshwork to the next. Although in paraffin sections, the distribution of red blood cells in the meshwork in the eye with congenital glaucoma indicated that potential drainage pathways were present throughout, in flat preparations there was a very marked reduction in the number of large spaces seen in the outer third of the meshwork in the region of Schlemm's canal. At the same time the number of preparations showing large numbers of small vacuoles in the cytoplasm was greater. These observations suggest that the incomplete differentiation of the angle, indicated by the failure of the uveal fibres to lengthen, has additional manifestations throughout the meshwork which are probably of considerable significance in producing obstruction to aqueous outflow. The meshwork in the eye with congenital glaucoma appeared to be potentially capable of further differentiation and it may be that goniotomies and other surgical procedures make further development possible by reducing intra-ocular pressure.

\section{Summary}

An examination of flat preparations of the trabecular meshwork in infants has shown large numbers of intercommunicating spaces and vacuoles which vary in size from 1 to $20 \mu$. The evidence suggests that these spaces represent stages in the development of drainage channels through the meshwork. In a case of congenital glaucoma there appeared to be a significant reduction in the number of larger spaces in the meshwork and an increase in the number of smaller spaces. These manifestations of a failure in development in the outer layers would be expected to result in an impairment of aqueous outflow and an increase in intra-ocular pressure.

We are indebted to Dr. A. J. Elliot and Dr. E. F. Raynor for an opportunity to examine the eye with congenital glaucoma.

\section{REFERENCES}

Allen, L., Burian, H. M., and Braley, A. E. (1955). A.M.A. Arch. Ophthal., 53, 782.

AshtoN, N. (1960). In "Glaucoma: Trans. IV Conf., 1959", p. 91. Josiah Macy, Jr., Foundation, New York.

BECKER, B. (1959). Ibid., p. 227.

Garron, L. K., and FeENEY, M. L. (1959). A.M.A. Arch. Ophthal., 62, 966.

HOLMBERG, A. (1959). Ibid., 62, 956.

Leeson, T. S., and SPEAKman, J. S. (1961). Acta anat. (Basel), 46, 363.

MAUMENEE, A. E. (1958). Trans. Amer. ophthal. Soc., 56, 507. (1962). Ibid., 60, 140.

SPEAKMAN, J. S. (1959). Brit. J. Ophthal., 43, 139.

(1960). Ibid., 44, 513.

and LEESON, T. S. (1962). Ibid., 46, 321.

SondermanN, R. (1930). v. Graefes Arch. Ophthal., 124, 521.

Teng, C. C., Katzin, H. M., and CHI, H. H. (1957). Amer. J. Ophthal., 43, 193. 